\title{
Logopädie: Sprache nicht dem Zufall überlassen
}

\author{
Am 6. März 2009 ist der Europäische Tag der Logopädie. Der Deutschschweizer \\ Logopädinnen- und Logopädenverband DLV begeht diesen Tag unter dem \\ Motto «Sprache nicht dem Zufall überlassen» und widmet sich insbesondere \\ dem Thema «Frühzeitige Intervention».
}

DLV Deutschschweizer Logopädinnen- und Logopädenverband
Korrespondenz:

DLV Deutschschweizer Logopädin-

nen- und Logopädenverband

Stampfenbachstrasse 142

CH-8006 Zürich

www.logopaedie.ch
Isaac Newton und Bruce Willis sind bzw. waren Stotterer. Auch bei manch anderer prominenten Persönlichkeit gilt ein Sprechfehler als charmantes Markenzeichen. Im normalen Alltag hingegen schafft fehlende Sprachkompetenz Probleme. Der 6. März 2009 steht deshalb unter dem Motto «Sprache nicht dem Zufall überlassen». Besonderes Augenmerk gilt der frühzeitigen Intervention - bei Kleinkindern, Schulkindern und Erwachsenen. Denn wenn es um logopädische Massnahmen bei Sprach-, Sprech- und Schluckstörungen geht, ist Abwarten keine Option: Zu schwerwiegend können Folgeerscheinungen aufgrund von verpassten Interventionen sein. Nicht nur für die Betroffenen, auch für ihre Familien, ihr Umfeld und schliesslich für die ganze Gesellschaft. Um eine möglichst hohe Effektivität der Therapie zu gewährleisten, arbeiten Logopädinnen und Logopäden eng mit anderen Berufsgruppen in Spitälern, Heimen und Schulen zusammen. Besonderen Stellenwert hat dabei der Austausch mit Ärztinnen und Ärzten.

\section{Kleinkinder: Sprachkompetenz stärkt Persönlichkeit}

Die Sprache beginnt mit vorsprachlichen Fähigkeiten wie Saugen und der Entwicklung des symbolischen Verständnisses. Deshalb beginnt eine logopädische Intervention schon häufig vor dem ersten gesprochenen Wort des Kindes: Neue Ansätze in Diagnose und Therapie erlauben früh, das Kind in seiner Sprachentwicklung zu unterstützen und die Freude an der Kommunikation zu wecken. Wird der Spracherwerb in einer sensiblen Phase dem Zufall überlassen, sind Probleme vorprogrammiert.

Grosse Bedeutung kommt der professionellen Beratung zum Beispiel von Eltern, Kinderärzten oder Hebammen zu. Risikokinder werden so frühzeitig erfasst, was eine entsprechende Therapie ermöglicht.

\section{Schulkinder: soziale Netze erhalten}

Sprechen und verstehen heisst am sozialen Geschehen teilnehmen, die Welt verstehen, abstra- hieren können. Fehlende sprachliche Kompetenz hingegen hemmt die kognitive, psychosoziale und kommunikative Entwicklung empfindlich. Frühe Intervention bedeutet frühzeitiges Auffangen von sprachlichen Schwierigkeiten im Leseund Schreiblernprozess im interdisziplinären Rahmen. Sie ist entlastend für die Lehrpersonen, die Eltern und das Umfeld.

\section{Erwachsene: Wiedereingliederung ins Leben}

Wenn Erwachsene ihre Sprache ganz oder teilweise verlieren, ist eine rasche und hohe Therapieintensität von grosser Bedeutung. Bei einer durch Hirnschädigung hervorgerufenen Aphasie kann die spontane Erholung des Gehirns durch frühzeitige Logopädie-Therapie optimal genutzt werden. Von Anfang an können so Ressourcen mobilisiert und hinderliche Verhaltensweisen gehemmt werden. Gleichzeitig sollen Familie und Bekannte frühzeitig über die Krankheit informiert und mögliche Unterstützungsmöglichkeiten aufgezeigt werden. Auch bei Erwachsenen ist interdisziplinäre Arbeit angezeigt. Damit werden die Chancen auf eine möglichst rasche Wiedereingliederung und die Partizipation im alltäglichen Leben erhöht

\section{Logopädie: so früh wie möglich}

Kinder, die noch nicht sprechen oder nicht verstanden werden, Kinder, deren schulische Leistungen aufgrund fehlender Lese- und Rechtschreibkompetenz unter ihren Fähigkeiten liegen, Erwachsene, die nach einem Unfall den Weg zurück in die Berufswelt suchen: Jeder kennt sie und ist somit betroffen. Dank der engen Zusammenarbeit der Fachkräfte ist es möglich, individuelle, effiziente und wirtschaftliche Lösungen zu finden. Damit Sprache nicht dem Zufall überlassen wird: Tag der Logopädie, 6. März 2009. 